\title{
Gut phages at the centre
}

The human gut microbiome comprises a complex microbial community that contains bacteria, archaea, fungi and other microbial eukaryotes, as well as viruses. Whereas the bacterial community has been well studied and is well described, the human gut virome has been studied to a lesser extent. Previous studies have shown that the virome is mainly composed of phages (phage virions or integrated into their hosts as prophages). Indeed, phages are the most abundant members of the microbiota. In other ecosystems, phages were shown to have many important roles in microbial ecology, population dynamics, physiology and evolution. However, much remains to be learnt about the composition, function and dynamics of the gut phage community and the possible contribution of phages to human health and disease. Two new studies shed some more light on these open questions and report the positive correlation between microbiome diversity and phage diversity, and the link between phage expansion and intestinal inflammation.

In the first study, Ley and colleagues examined the viromes of 21 monozygotic twin pairs (thus removing host genetic relatedness as a variable) to examine the relationship between the diversity of the microbiome and the diversity

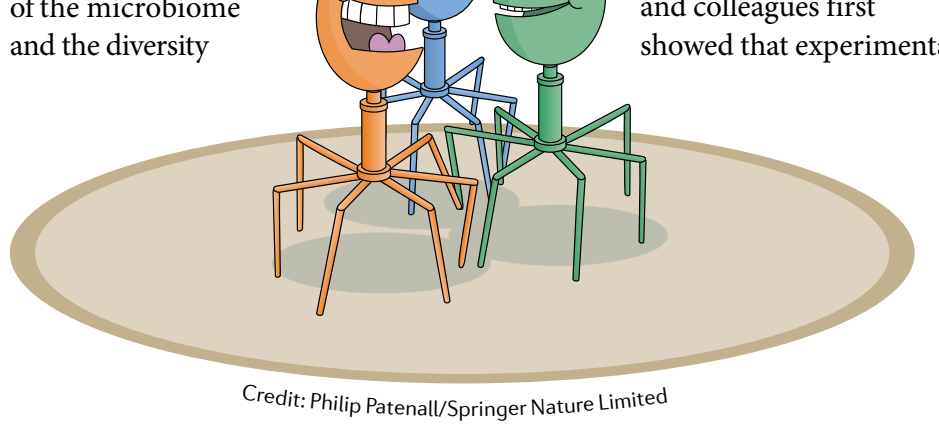

phage therapy with phages against adherent invasive Escherichia coli isolated from the human microbiota significantly reduced colonization by those carcinogenic bacteria and prevented tumour growth and mortality in mice. However, the authors noticed that both innate and adaptive immune pathways were upregulated in phage-treated animals compared with control animals.

To test whether phages directly stimulate the mammalian immune system, they administered the purified phage cocktail or a vehicle control to germ-free mice and found that phage treatment resulted in immune cell expansion in the gut. Moreover, they were able to show that phage DNA recognition by dendritic cells stimulated the production of the cytokine interferon- $\gamma$ (IFN $\gamma)$ by $\mathrm{CD} 4{ }^{+} \mathrm{T}$ cells. The authors also showed that immune activation was mediated by Toll-like receptor 9 (TLR9), as incubation of purified phage DNA with dendritic cells that lacked TLR9 failed to stimulate IFN $\gamma$ production.

Finally, using a phage cocktail that contained Caudovirales phages, the authors reported that increased phage abundance exacerbated intestinal colitis in a manner dependent on TLR9 and IFN $\gamma$ production, as indicated by the finding that TLR9-deficient mice were protected from phageenhanced colitis and exhibited reduced immune responses.

In sum, the study provides evidence that changes in gut phage communities can directly aggravate intestinal disease.

\section{Andrea Du Toit}

ORIGINAL ARTICLES Moreno-Gallego, J. L., Chou, S.-P. et al. Virome diversity correlates with intestinal microbiome diversity in adult monozygotic twins. Cell Host Microbe https:// doi.org/10.1016/j.chom.2019.01.019 (2019)| Gogokhia, L. et al. Expansion of bacteriophages is linked to aggravated intestinal inflammation and colitis. Cell Host Microbe https://doi.org/10.1016/ j.chom.2019.01.008 (2019) FURTHER READING Khan Mirzaei, M. $\&$ Maurice, C. F. Ménage à trois in the human gut: interactions between host, bacteria and phages. Nat. Rev. Microbiol. 15, 397-408 (2017) 